\title{
Efecto del portainjerto en el índice y densidad estomática de pimiento morrón Capsicum annuum var. annuum
}

\section{Effect of the rootstock on the stomatal index and density of bell pepper Capsicum annuum var. annuum}

\author{
Neymar Camposeco-Montejo ${ }^{1 *}$, Valentín Robledo-Torres ${ }^{1}$, Francisca Ramírez-Godina ${ }^{2}$, Luis Alonso \\ Valdez-Aguilar ${ }^{1}$, Marcelino Cabrera-de-la-Fuente ${ }^{1}$, Rosalinda Mendoza-Villareal ${ }^{1}$ \\ ${ }^{1}$ Departamento de Horticultura, Universidad Autónoma Agraria Antonio Narro. Calzada Antonio Narro 1923, CP. 25315. \\ Saltillo, Coahuila, México. \\ ${ }^{2}$ Departamento de Fitomejoramiento, Universidad Autónoma Agraria Antonio Narro. Calzada Antonio Narro 1923, CP. 25315. \\ Saltillo, Coahuila, México. \\ *Autor de correspondencia: neym 33k@hotmail.com
}

Nota científica recibido: 02 de julio de 2017 aceptado: 22 de febrero de 2018

RESUMEN. El objetivo fue determinar el efecto del portainjerto y del injerto en el índice y la densidad estomática del chile pimiento. Los tratamientos fueron, pimiento injertado y sin injertar, con cinco repeticiones. La densidad e índice estomático, largo y ancho de estomas se determinaron con un microscopio Carl Zeeis con cámara integrada y software de medición AxionVisionRel.4.8. Se encontraron diferencias significativas en densidad e índice estomático adaxial y abaxial, superando el pimiento injertado al sin injertar en $26.20,21.07$ y $8.74 \%$, respectivamente. El pimiento sin injertar superó en $8.51 \%$ al injertado en densidad de células epidérmicas abaxiales, mientras que el largo y ancho de estomas del pimiento injertado superó en 8.64 y $11.22 \%$ al sin injertar. El portainjerto incrementó el índice y la densidad estomática del haz y del envés, tamaño de estomas y células epidérmicas del envés, modificando el comportamiento fisiológico de la planta.

Palabras clave: Injerto, estomas, transpiración, morfología foliar, dióxido de carbono

ABSTRACT. The aim was to determine the effect of the rootstock and graft on the stomatal index and density of pepper chili. The treatments were grafted and ungrafted pepper, with five replicates. Stomatal density, index, length and width were determined with a Carl Zeeis microscope with an integrated camera and AxionVisionRel.4.8 measuring software. Significant differences were found in adaxial and abaxial stomatal density and index, with the grafted pepper surpassing the ungrafted pepper by $26.20,21.07$ and $8.74 \%$, respectively. The ungrafted pepper surpassed the grafted pepper by $8.51 \%$ in density of abaxial epidermal cells, while the grafted pepper surpassed the ungrafted pepper by 8.64 and $11.22 \%$ in stomatal length and width, respectively. The rootstock increased the stomatal index and density of the surface and underside, as well as the size of stomata and epidermal cells of the underside, thereby modifying the physiological behavior of the plant.

Key words: Graft, stomata, transpiration, foliar morphology, carbon dioxide

\section{INTRODUCCIÓN}

El conocimiento de las características anatómicas y morfológicas de las hojas de un cultivo es de gran importancia, debido a que realizan las funciones de intercambio gaseoso entre las hojas y la atmosfera. El intercambio de gases general- mente se realiza en los estomas de la epidermis, cuya función principal es la asimilación de $\mathrm{CO}_{2}$ y la pérdida de agua por transpiración, absorbiendo nutrimentos minerales por flujo de masas bajo condiciones ambientales cambiantes que ejercen presión sobre un determinado cultivo (Barrientos et al. 2003, Sánchez y Aguirreolea 2008). Las 


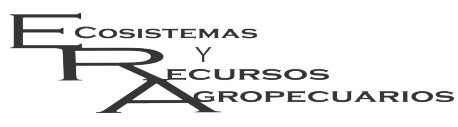

Camposeco-Montejo et al.

Densidad Estomática En Pimiento Morrón

Ecosist. Recur. Agropec.

5(15):555-561,2018

condiciones ambientales como radiación solar, temperatura, humedad relativa, humedad del suelo o sustrato, velocidad del viento, concentración de $\mathrm{CO}_{2}$, nutrientes minerales, no solo influyen sobre la difusión y transpiración, sino también en la apertura y cierre estomático de la superficie foliar a través de los que pasa el $\mathrm{CO}_{2}$ y el agua que puede ser de hasta el $95 \%$ del total que utiliza la planta (Salisbury y Ross 2000, Naizaque et al. 2014).

Por otra parte el injerto, consiste en unir una parte de una planta a otra que ya está asentada, resultando un individuo autónomo, formado por dos plantas; el portainjerto que genéticamente contiene genes de resistencia o tolerancia a estrés biótico o abiótico (Aidoo et al. 2017, Zhao et al. 2011). Mientras que el injerto o variedad comercial es una porción de tallo o yema que se fija al portainjerto para que se desarrollen ramas, hojas, flores y frutos (Hartmann et al. 1997). Cultivos como sandía, melón, pepino, tomate entre otros son comúnmente injertados (Sakata et al. 2008), originalmente el propósito de la técnica de injerto en cultivos hortícolas era evadir las enfermedades causadas por patógenos del suelo (Louws et al. 2010), actualmente también se utiliza para evitar problemas de estrés abiótico (Kumar et al. 2016), incrementar rendimientos y extender tiempo de cosecha (Martínez-Ballesta et al. 2010). Además permiten reducir las aplicaciones de fertilizantes e incrementar la calidad de los frutos (Schwarz et al. 2010). El uso de portainjertos resistentes, en combinación con prácticas de manejo integrado de plagas y enfermedades, representa una alternativa viable, que permiten reducir el uso de agroquímicos (King et al. 2010). En México, se reportan injertos en chile tipo ancho (García et al. 2010), jalapeños, chilacas y cayenne (Osuna et al. 2012), con incrementos del rendimiento de hasta 50\% (Sánchez et al. 2015, Penella et al. 2017). En tomate injertado también se han reportado incrementos de rendimiento de hasta 35\% (Álvarez 2012).

La tendencia del incremento en el uso de los injertos, genera nuevas interrogantes a las que hay que dar respuesta, con el fin de optimizar las unidades de producción para obtener mayores rendimientos y calidad (Huang et al. 2015). Aunque el rendimiento agronómico de un cultivo está determinado por el número de frutos cosechados por unidad de área y su tamaño individual, estos a su vez dependen de la arquitectura, anatomía, funciones fisiológicas y metabólicas de la planta (Peil y Galvez 2004). Al respecto, Ayala et al. (2010) señalan que al incrementar la densidad estomática de las hojas en los injertos, estos incrementan su tasa de asimilación de $\mathrm{CO}_{2}$, la transpiración y conductancia estomática; además de la eficiencia en el uso del agua y la tolerancia de las plantas al estrés por salinidad (Salas et al. 2001); lo que influye en la eficiencia fisiológica de las hojas para asimilar más $\mathrm{CO}_{2}$ y transformarlo en asimilados (Ayala-Tafoya et al. 2015). Aunque se sabe de los beneficios de injertar las plantas, también es de gran importancia conocer el ajuste y las modificaciones epidérmicas de las hojas de los cultivos, dado que es donde se realiza el intercambio de gases para el proceso fotosintético que genera los fotoasimilados para traslocarlos a los frutos y demás órganos de demanda de la planta. Por lo anterior el objetivo fue determinar el efecto del injerto y el portainjerto en el índice y la densidad estomática del chile pimiento morrón.

\section{MATERIALES Y MÉTODOS}

Se utilizó el genotipo Foundation RZ F1 como portainjerto, y como injerto el híbrido Bambuca F1 de color amarillo tipo blocky. El establecimiento del cultivo para la evaluación de índice estomático (IE) y densidad estomática (DE) se realizó en las instalaciones del invernadero de mediana tecnología del Departamento de Horticultura de la Universidad Autónoma Agraria Antonio Narro (UAAAN), en Saltillo, Coahuila, México; ubicada a $25^{\circ} 21^{\prime}$ 24" LN y $101^{\circ} 02^{\prime}$ 05" LO, con altitud de 1762 msnm, precipitación media de $400 \mathrm{~mm}$, temperatura media anual entre 12 y $18{ }^{\circ} \mathrm{C}$, y clima $\mathrm{BS}_{0} \mathrm{k}\left(\mathrm{x}^{\prime}\right)(\mathrm{e})$.

\section{Establecimiento en campo y manejo del cultivo}

Se utilizaron plantas de pimiento injertadas y plantas sin injertar. Se estableció el cultivo en 


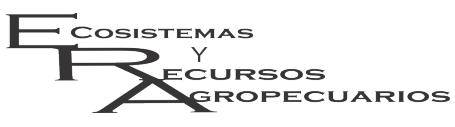

macetas de $10 \mathrm{~L}$ con sustrato peat moos y perlita en proporción 75:25\%, en el ciclo Primavera-Verano de 2016, la distancia entre plantas dentro de hileras fue de $30 \mathrm{~cm}$ y de $1.80 \mathrm{~m}$ entre hileras, cada planta se manejó a dos tallos, bajo un arreglo experimental completamente al azar con dos tratamientos y cinco repeticiones, cada repetición con cinco plantas útiles, donde el tratamiento control fue el pimiento sin injertar (Bambuca F1) comparado con pimiento injertado (Foundation F1 + Bambuca F1). Se utilizó la solución nutritiva propuesta por Steiner (1966), que se aplicó vía riego por espagueti a lo largo del ciclo de cultivo, la cual fue del $50 \%$ al inicio del cultivo, $75 \%$ a los $30 \mathrm{~d}$ después del trasplante y de $100 \%$ al inicio de la floración. Para la prevención y control de plagas (mosca blanca, trips, paratrioza) se realizaron aplicaciones semanales de Spirotetramat al 15.3\%, Spiromesifen al 23.1\%, Imidacloprid $17 \%+$ cylfutrin $12 \%$ a razón de $1 \mathrm{ml}$ $\mathrm{L}^{-1}$ y metomilo $90 \%$, a razón de $1 \mathrm{~g} \mathrm{~L}^{-1}$.

\section{Toma de muestras epidérmicas}

La toma de fotografías para contabilizar y realizar el análisis estomático se realizó en el laboratorio de Citogenética del Departamento de Fitomejoramiento de la UAAAN, las hojas se colectaron $60 \mathrm{~d}$ después del trasplante, al inicio de la floración, y se realizaron impresiones epidérmicas en hojas completamente maduras y con la misma orientación de cada planta útil, por medio de la aplicación de barniz transparente en un área de aproximadamente $2 \mathrm{~cm}^{-2}$ en la parte media de las venas secundarias, en el haz y el envés de las hojas, después de que el barniz se secó, se removio la capa de barniz con una cinta adhesiva transparente, que se colocó en un portaobjetos de vidrio. Se tomaron 3 fotografías al azar de cada impresión epidérmica, para un total de 15 fotografías por repetición y tratamiento, con un microscopio Carl Zeis con cámara integrada (Pixera Winder Pro), con el objetivo de 10X, en las que se determinó la densidad estomática (DE) del haz y envés, contabilizando los estomas existentes en el área de la fotografía $\left(0.3965 \mathrm{~mm}^{-2}\right)$ de acuerdo a la siguiente formula $\mathrm{DE}=$ Número de estomas/área de la fotografía
Camposeco-Montejo et al. Densidad Estomática En Pimiento Morrón Ecosist. Recur. Agropec. 5(15):555-561,2018

$\left(0.3965 \mathrm{~mm}^{-2}\right)$, para obtener el número de estomas por $1 \mathrm{~mm}^{-2}$. Mientras que para el índice estomático las fotografías se tomaron con el objetivo de $40 \mathrm{X}$ del haz y envés, para luego calcular el índice estomático (IE) con la siguiente expresión, IE = (DE / (DE + $\mathrm{DCE}))^{*} 100$ donde; $\mathrm{DE}=$ densidad estomática y DCE $=$ densidad de células epidérmicas (Salisbury y Roos 2000), el área de la fotografía de $0.0240 \mathrm{~mm}^{-2}$ para luego ajustar a $1 \mathrm{~mm}^{-2}$. Con las fotografías tomadas con el objetivo de $40 \mathrm{X}$ se midió el largo y ancho de estomas, expresado en micrómetros, para lo cual se utilizó el software Axion Vision Rel.4.8.

Para el análisis estadístico, se realizo una prueba de $\mathrm{T}$ simple con una confiabilidad del $95 \%$, con el software estadístico Minitab16, donde los tratamientos fueron el pimiento morrón injertado y pimiento morrón sin injerto.

\section{RESULTADOS Y DISCUSIÓN}

La prueba de $t$ realizada para densidad estomática del haz, índice estomático del haz e índice estomático del envés, muestra diferencias significativas $(p \leq 0.01)$ entre tratamientos, mientras que para la densidad de células epidérmicas del envés se tuvieron diferencias significativas al $p \leq 0.05$ (Tabla 1). Lo que coincide con lo reportado por Peralta et al. (2016) quienes reportan diferencias estadísticas significativas en el índice y densidad estomática del haz y el envés en pepino injertado. Mientras que Ayala et al. (2010) no encontraron diferencias estadísticas en la densidad de células del envés entre plantas injertadas y no injertadas de Aguacate Hass. Para las variables de densidad estomática del envés y densidad de células epidérmicas del haz, no se encontraron diferencias significativas, lo que difiere con González et al. (2017) quienes reportan diferencias significativas en sandia injertada y $\sin$ injertar en la densidad estomática.

Para las plantas de pimiento injertado y sin injertar, la densidad estomática del haz, índice estomático del haz y del envés, tuvo los mayores valores el pimiento injertado, con valores de 26.20 , 21.07 y $8.74 \%$ superiores a los valores obtenidos en el tratamiento sin injertar, incrementos que son 
Camposeco-Montejo et al.

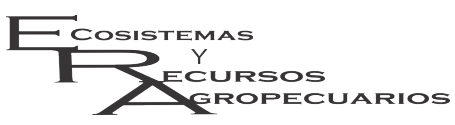

Densidad Estomática En Pimiento Morrón

Ecosist. Recur. Agropec.

5(15):555-561,2018

Tabla 1. Prueba de $t$ simple, de tres caracteres epidérmicos foliares evaluados en pimiento injertado y sin injertar.

\begin{tabular}{|c|c|c|c|c|c|c|}
\hline \multirow[b]{2}{*}{ Tratamientos } & \multicolumn{3}{|c|}{$\mathrm{Haz}$} & \multicolumn{3}{|c|}{ Envés } \\
\hline & $\begin{array}{c}\mathrm{DE} \\
\left(\text { Estomas } \mathrm{mm}^{-2} \text { ) }\right.\end{array}$ & $\begin{array}{l}\mathrm{IE} \\
(\%)\end{array}$ & $\begin{array}{c}\text { DCE } \\
\left(\text { Células } \mathrm{mm}^{-2} \text { ) }\right.\end{array}$ & $\begin{array}{c}\mathrm{DE} \\
\left(\text { Estomas } \mathrm{mm}^{-2} \text { ) }\right.\end{array}$ & $\begin{array}{c}\text { IE } \\
(\%)\end{array}$ & $\begin{array}{c}\text { DCE } \\
\left(\text { Células } \mathrm{mm}^{-2} \text { ) }\right.\end{array}$ \\
\hline Bambuca & $30.15 \pm 2.86$ & $8.59 \pm 0.84$ & $323.14 \pm 27.5$ & $152.78 \pm 22.4$ & $22.76 \pm 2.15$ & $519.44 \pm 48.1$ \\
\hline Foundation+Bambuca & $38.05 \pm 5.63$ & $10.4 \pm 1.31$ & $328.70 \pm 23.9$ & $157.6 \pm 27.4$ & $24.75 \pm 3.22$ & $478.7 \pm 42.6$ \\
\hline Valor de $\mathrm{P}$ & 0.000 & 0.000 & 0.560 & 0.602 & 0.059 & 0.021 \\
\hline
\end{tabular}

$\mathrm{p} \leq 0.05, \mathrm{DE}=$ densidad estomática, $\mathrm{IE}=$ índice estomático, $\mathrm{DCE}=$ densidad de células epidérmicas.

similares a lo reportado por Cañizares et al. (2003) y Ayala et al. (2010), pero contrastan con lo reportado por González et al. (2017) para sandía y con Peralta et al. (2016) para pepino, quienes señalan que en las plantas injertadas se reduce la densidad estomática del haz y envés, y el índice estomático del haz. La densidad de células del envés del pimiento sin injertar superó en $8.51 \%$ al pimiento injertado, lo que indica que las células se hacen más grandes con el portainjerto, lo que difiere con lo reportado por Ayala et al. (2010) quienes indican que las plantas injetadas tienen mayores valores.

Los incrementos en densidad estomática, índice estomático y densidad de células epidérmicas se debió probablemente al vigor que le confieren el portainjerto a la variedad injertada, ya que se correlaciona de forma directa con la tasa de asimilación neta de $\mathrm{CO}_{2}$, la tasa de respiración y la conductancia estomática al tiempo que se reduce la resistencia estomática como lo mencionan Ayala et al. (2010), lo que influye con la eficiencia fisiológica de las hojas para asimilar $\mathrm{CO}_{2}$ y transformarlo en asimilados que se transportan a los sitios de demanda, lo que confiere mayor eficiencia productiva a las plantas. Además de la eficiencia en el uso del agua y la tolerancia de las plantas al estrés por salinidad (Salas et al. 2001). Al respecto Naizaque et al. (2014) señalan que a medida que aumenta el número de estomas y la temperatura en las hojas, se incrementa la tasa de transpiración, lo que impacta en la absorción y transporte nutrimental. Las plantas ajustan y actualizan su anatomía, fisiología y metabolismo de acuerdo a las modificaciones que sufren y las condiciones del medio ambiente, que ejerce presión en un momento determinado, cambiando sus ritmos de funcionamiento, afectando su crecimiento y desarrollo (Hernando et al. 2017).
El pimiento morrón es una especie anfiestomática, debido a que presenta estomas en las dos caras de las hojas, con mayor número de estomas en el envés que supera al haz en un $500 \%$. Los resultados muestran los efectos que ejerce el portainjerto utilizado, que induce modificaciones de las características micromorfológicas foliares del pimiento morrón.

Para el largo y ancho de estomas del haz y del envés de las hojas de pimiento (Figura 1 ), se encontró que el largo y ancho de los estomas del envés fueron estadísticamente significativos (Tabla 2), siendo mayores en el pimiento injertado, que superó en 8.64 y $11.22 \%$ al pimiento sin injertar. Aunque no se encontraron diferencias estadísticas para las mismas variables en el haz de las hojas, el pimiento injertado superó en 3.48 y $5.07 \%$ al pimiento sin injertar. Lo que difiere de lo reportado por González et al. (2017) para sandía y Peralta et al. (2016) para pepino, quienes indican que el largo y ancho de los estomas del haz y envés permanece sin modificaciones. Lo que indica que los estomas son más grandes con el uso del portainjerto, lo que se puede deber al vigor que le confiere el portainjerto a la variedad o la mayor hidratación celular por la mayor eficiencia del sistema radicular, lo que impacta en un mayor tamaño celular en el envés de las hojas, lugar donde se realiza el mayor intercambio gaseoso en las hojas y la atmosfera, lo que se correlaciona de forma positiva con el incremento de la tasa de transpiración y asimilación de nutrientes por flujo de masas e influye en la eficiencia fisiológica de las hojas para asimilar más $\mathrm{CO}_{2}$ y transformarlos en asimilados (Salas et al. 2001, Naizaque et al. 2014).

El índice y densidad estomática del haz y del envés de las hojas se incrementó con el uso del portainjerto. Lo que impacta en el incremento de la 

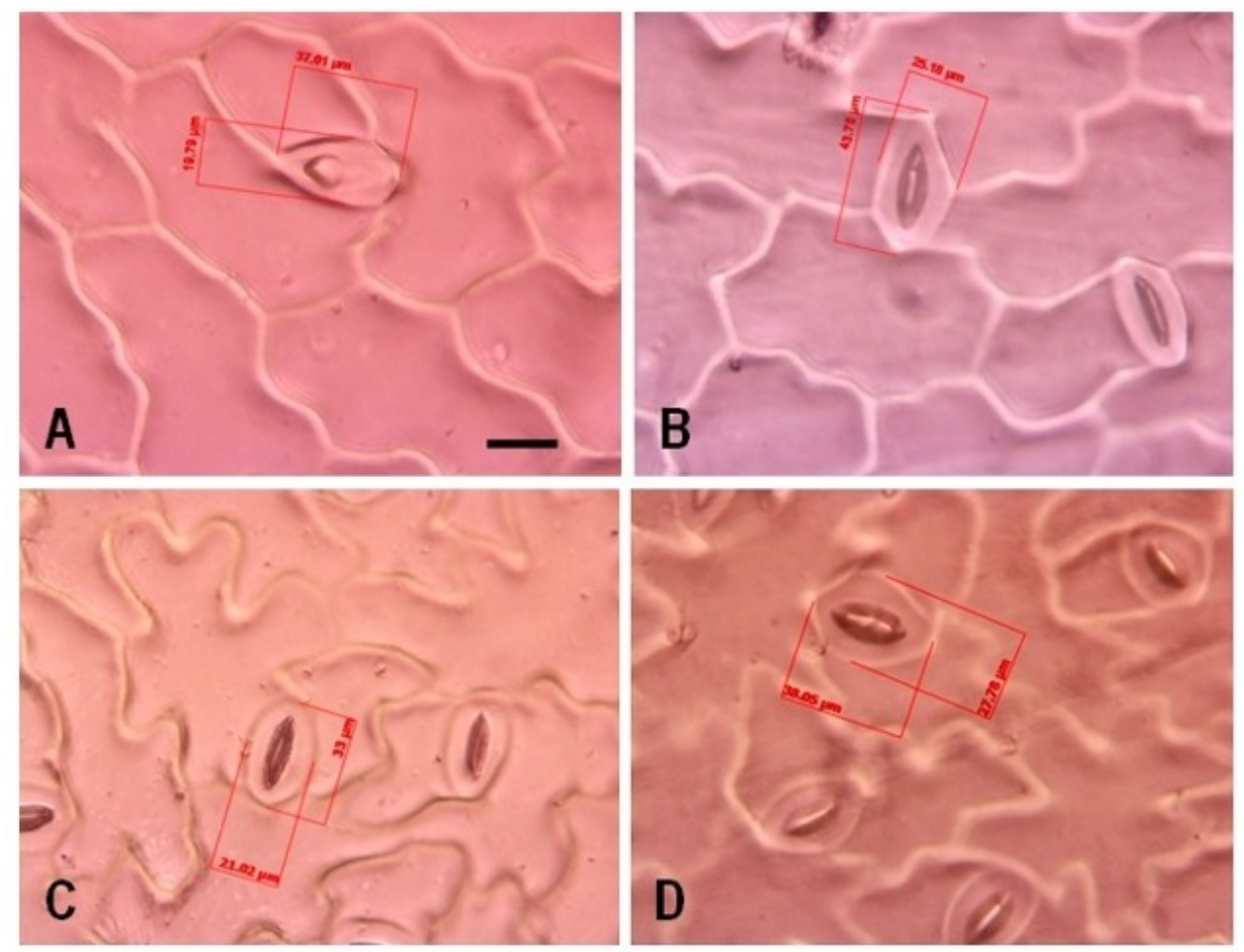

Figura 1. Impresiones de la epidermis de hojas de pimiento. Largo y ancho de estomas del haz (A) sin injerto, (B) con injerto. Estomas del envés (C) sin injerto y (D) con injerto $40 \mathrm{X}$, escala $20 \mu \mathrm{m}$.

Tabla 2. Prueba de $\mathrm{T}$ simple, de dos caracteres estomáticos evaluados en pimiento injertado y sin injerto.

\begin{tabular}{ccccc}
\hline \multirow{2}{*}{ Tratamientos } & \multicolumn{2}{c}{ Haz } & \multicolumn{2}{c}{ Envés } \\
\cline { 2 - 5 } & Largo & $\begin{array}{c}\text { Ancho } \\
(\mu \mathrm{m})\end{array}$ & $\begin{array}{c}\text { Largo } \\
(\mu \mathrm{m})\end{array}$ & $\begin{array}{c}\text { Ancho } \\
(\mu \mathrm{m})\end{array}$ \\
\hline Bambuca & $38.98 \pm 2.46$ & $21.28 \pm 2.97$ & $34.81 \pm 2.77$ & $24.77 \pm 1.92$ \\
Foundation+Bambuca & $40.34 \pm 2.23$ & $22.36 \pm 2.13$ & $37.82 \pm 3.37$ & $27.55 \pm 3.34$ \\
Valor de P & 0.124 & 0.261 & 0.013 & 0.011 \\
\hline $\mathrm{p} \leq 0.05, \mu \mathrm{m}=$ (micrómetros). & & &
\end{tabular}

tasa de asimilación de $\mathrm{CO}_{2}$, la tasa de transpiración y asimilación de nutrientes, la conductancia estomática y la reducción de la resistencia estomática como lo describe Ayala et al. (2010). El portainjerto modificó la lámina foliar de pimiento, lo que modifica el intercambio gaseoso, la tasa de transpiración y fotosintética.

\section{LITERATURA CITADA}

Aidoo MK, ShermanT, Ephrath JE, Fait A, Rachmilevitch S, Lazarovitch N (2017) Grafting as a method to increase the tolerance response of bell pepper to extreme temperatures. Vadose Zone Journal 17. Doi: 10.2136/vzj2017.01.0006.

Álvarez HJC (2012) Comportamiento agronómico e incidencia de enfermedades en plantas de tomate (Solanum lycopersicum L.) injertadas. Acta Agronómica 61: 117-125.

Ayala-Tafoya F, Yañez-Juárez MG, Partida-Ruvalcaba L, Ruiz-Espinosa FH, Campos-García H, Vásquez- 
Martínez T et al. (2015) Producción de pepino en ambientes diferenciado por mallas de sombreo fotoselectivo. Información Tecnica Económica Agraria 111: 3-17.

Ayala AJ, Barrientos PAF, Colinas LMT, Sahagún CJ, Reyes AJC (2010) Relaciones injerto-interinjerto y características anatómicas y fisiológicas de la hoja de cuatro genotipos de aguacate. Revista Chapingo Serie Horticultura 16: 147-154.

Barrientos PAF, Borys MW, Trejo C, López LL (2003) Índice y densidad estomática foliar en plántulas de tres razas de aguacatero. Revista Fitotecnia Mexicana 26: 285-290.

Cañizares A, Sanabria ME, Rodríguez DA, Perozo Y (2003) Características de los estomas, índice y densidad estomática de las hojas de lima Tahití (Citrus latifolia Tanaka) injertada sobre ocho patrones cítricos. Revista UDO Agrícola 3: 59-64.

García RMA, Chiquito AE, Loeza LPD, Godoy HH, Villordo PE, Pons HJL, et al. (2010) Producción de chile ancho injertado sobre criollo de Morelos 334 para el control de Phytophthora capsici. Agrociencia 44: 701-709.

González GH, Ramirez GF, Ortega OO, Benavides MA, Robledo TV, Cabrera de la FM (2017) Use of chitosanPVA Hidrogels with copper nanoparticles to improve the growth of grafted watermelon. Molecules 22: $1-9$.

Hartmann HT, Kester DE, Davies FT, Geneve RL (1997) Plant propagation. Ed. Prentice Hall. USA. 873p.

Hernando CE, Romanowski A, Marcelo J, Yanovsky (2017) Transcriptional and post-transcriptional control of the plant circadian gene regulatory network. Biochimica et Biophysica Acta (BBA) - Gene Regulatory Mechanisms 1860: 84-94

Huang W, Liao S, Haiyan L, Khaldun ABM, Wang Y (2015) Characterization of the growth and fruit quality of tomato grafted on a woody medicinal plant, Lycium chínense. Scientia Horticulturae 197: 447-453.

King SR, Davis AR, Zhang X, Crosby K (2010) Genetics, breeding and selection of rootstock for solanaceae and cucurbitaceae. Scientia Horticulturae 127: 106-111.

Kumar P, Rana S, Sharma P, Singh A, Updhyay SK (2016) Evaluation of chilli and brinjal rootstocks for growth, yield and quality of bell pepper (Capsicum annuum L. Var. grossum Sendt.) under protected conditions. Agricultural Research Journal 53: 180-183.

Louws JF, Rivard LC, Kubota C (2010) Grafting fruiting vegetables to manage soilborne pathogens, foliar pathogens, arthropods and weeds. Scientia Horticulturae 127: 125-146.

Martínez-Ballesta C, Alcaraz LC, Muries B, Mota CC, Carvajal M (2010) Physiological aspects of rootstockscion interaction. Scientia Horticulturae 127: 112-118.

Naizaque J, García G, Fischer G, Melgarejo LM (2014) Relación entre la densidad estomática, la transpiración y las condiciones ambientales en feijoa (Acca sellowiana [O. Berg] Burret). Revista UDCA Actividad \& Divulgacion Cientifica 17: 115-121.

Osuna AP, Aguilar SP, Fernández PS, Godoy HH, Corral DB, Flores MJP, et al. (2012) Injertos en chiles tipo Cayene, jalapeño y chilaca en el noroeste de Chihuahua, México. Revista Mexicana de Ciencias Agrícolas 3: 739-750.

Penella C, Nebauer SG, Lopez GS, Quiñonez A, Calatayud A (2017) Grafting pepper onto tolerant rootstocks: An environmental-friendly technique overcome water and salt stress. Sientia Horticulturae 226: 33-41. 
Peralta MRM, Cabrera de la FM, Morelos MA, Benavides MA, Ramírez GF, Gonzales FJA (2016) Micromorfologia del pepino obtenido mediante injerto y desarrollado en dos sistemas de fertilización. Revista Mexicana de Ciencias Agrícolas 17: 3453-3463.

Peil RMN, Galvez JL (2004) Rendimiento de plantas de tomate injertadas y efecto de la densidad de tallos en el sistema hidropónico. Horticultura Brasileira 22: 265-270.

Sakata Y, Ohara T, Sugiyama M (2008) The history of melon and cucumber grafting in Japan. Acta Horticulturae 767: 217-228.

Salas JA, Sanabria ME, Reinaldo P (2001) Variación en el índice y densidad estomática en plantas de tomate (Lycopersicon esculentum Mill.) sometidas a tratamientos salinos. Bioagro 13: 99-104.

Sánchez CE, Torres GA, Flores CMA, Preciado RP, Marquez QC (2015) Uso de portainjerto sobre el rendimiento, calidad del fruto y resistencia a Phytophthora capsici Leonian en pimiento morrón. Revista Electrónica Nova Scientia 7: 227-244.

Sánchez DM, Aguirreolea J (2008) Transpiracion y control estomático. En: Azcon BJ, Talon M (Eds). Fundamentos de fisiología vegetal. McGraw-Hill Interamericana. Madrid, España. pp: 41-56.

Salisbury F, Ross C (2000) Fisiologia de las plantas. Volumen 1 Paraninfo-Thompson Learning. Madrid España. 305p.

Schwarz D, Rouphael Y, Colla G, Venema JH (2010) Grafting as a tool to improve tolerance of vegetables to abiotic stresses: thermal stress, wáter stress and organic pollutants. Scientia Horticulturae 127: 162-171.

Steiner AA (1966) The influence of chemical composition of a nutrient solution on the production of tomato plants. Plant and Soil 24: 454-466.

Zhao X, Ghuo Y, Huber DJ, Lee J (2011) Grafting effects on postharvest ripening and quality of 1methylcyclopropene-treated muskmelon fruit. Scientia Horticulturae 130: 581-587. 
